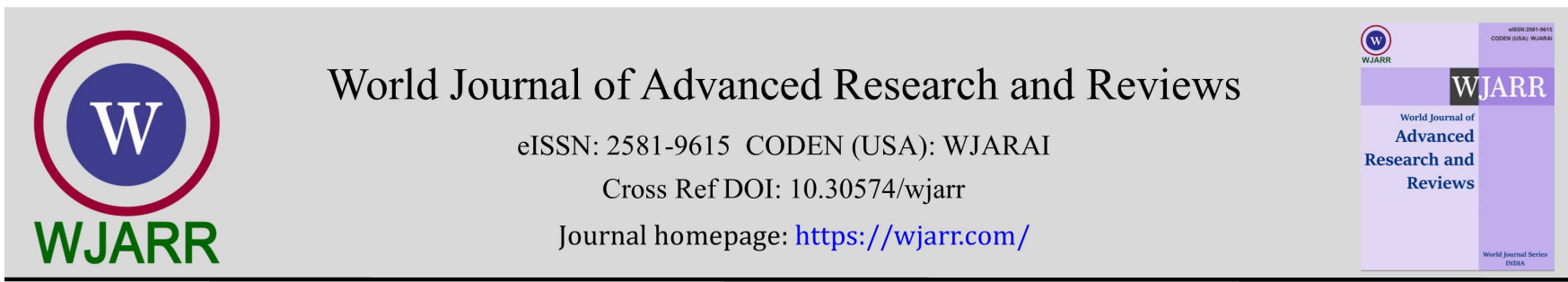

(RESEARCH ARTICLE)

Check for updates

\title{
Analysis of interventions and social work services in the Psychiatric Hospital "Sadik Dinci" Elbasan, Albania
}

\author{
Flutra Musta * and Monika Bogdanova \\ Department of Social Work, Faculty of Pedagogy, Sofia University "St. Kliment Ohridski", Sofia, Bulgaria.
}

World Journal of Advanced Research and Reviews, 2021, 10(01), 270-278

Publication history: Received on 17 March 2021; revised on 20 April 2021; accepted on 22 April 2021

Article DOI: https://doi.org/10.30574/wjarr.2021.10.1.0175

\begin{abstract}
Recent changes, especially after the 90s in Albania, have marked an important turn in the whole health system and even more so in the field of mental health. With health services, policies and social services were implemented to people with mental health problems, and it in this form that there was a need to bridge these policies and services to beneficiaries, such as social workers in mental health. Now the social worker is one of the key persons in the multidisciplinary team whose purpose is to identify, diagnose, treat, plan and integrate these people into society. The study aims is to identify the support and role of social work in persons with mental health disorders at Psychiatric Hospital "Sadik Dinçi" Elbasan, Albania and in the supported housing in this city. Qualitative method was used for conducting the study, and semi-structured interview with a focus group of 4 social workers of this hospital, 2 social workers of Elbasan Community Mental Health Center and 4 day-care ergo therapists located within the premises of the hospital was used as measuring instruments to this hospital. Also, a survey was conducted with 15 patients of the hospital. According to the study, the role of the social worker is well positioned in the field of mental health, but at the same time the study highlights the need for modern European interventions and models for integrating these individuals away from the walls of psychiatric hospitals, models where these individuals are supported and integrated in society.
\end{abstract}

Keywords: Mental health; Social policy; Social work; Intervention; Elbasan

\section{Introduction}

Mental health as a key component of overall health well-being is already one of the main pillars of health policies in Albania. All mental health services respect and promote the exercise and respect of the rights of users of these services, as they are mentioned and in the Law on Mental Health no. 44/2012 Albania [1].

Ministry of Health is the responsible authority for the development of mental health services with intent at promoting, preventing, diagnosing, treating and rehabilitating in the field of mental health. Ministry of Health supports activities and initiatives against discrimination, stigmatization and exclusion of persons with mental illness or disability and takes care of the full respect of their human rights. In the field of mental health, human resources development has been identified as one of the most priority issues in both the 2003 Policy Paper [2] and the Action Plan for the Development of Mental Health Services (2013 - 2020) [3]. The undertaken reform in the recent years in the field of mental health has led not only the change of services typology, but also the expand range of professionals involved in mental health services. Expanding the staff with new professionals, turning it into a multidisciplinary team composed not only of doctors and nurses, but also of psychologists, social workers, occupational therapists, etc. The establishment of new services previously unknown with community-base is also reflected in the unknown roles that professionals must play in these services, focused not only on treatment but also on prevention and rehabilitation. Also, during the cooperation of services and their professionals, was identified the need of updating the professional roles based on the experiences

${ }^{*}$ Corresponding author: Flutra Musta

Department of Social Work, Faculty of Pedagogy, Sofia University "St. Kliment Ohridski", Sofia, Bulgaria.

Copyright (C) 2021 Author(s) retain the copyright of this article. This article is published under the terms of the Creative Commons Attribution Liscense 4.0. 
of the best practices, as well as their enrichment with other professional roles present in these services. This category of services includes all those services that their focus is on the objectives and their functioning have the diagnosis, treatment and rehabilitation of persons with mental disorders, as well as the prevention of these disorders and the promotion of positive mental health. Based on the vision of the MH (Ministry of Health), as described and in the Policy Document [2,3], this network services should consist, but not be limited of services such as: Specialized outpatient services; Mental health services with community-base, such as community mental health centers, multidisciplinary mobile teams, day care centers, support homes for former chronic patients in psychiatric hospitals, support homes for psychotic patients, day care/recreation centers, etc.

Mental health services with beds, which should be provided near the mental health wards at regional hospitals, or near psychiatric hospitals covering the need for secondary and tertiary level care. Despite the MH's commitment to expand this network, based on existing specialized mental health services, this regulation will describe the main roles, objectives and functions of the services. Among these services is the provision of social services which support the process of using means to support usually individuals with chronic mental health disorders. Social services policies are addressed in a new mental health context, where adequate living conditions must be provided for patients with this disorder through economic and material assistance as well as giving them the opportunity to live in the community or in housing, protected or semi-protected. The purpose of social service interventions is to maintain and strengthen the health of clients by working with their social problems and in particular, by improving their relationships, adaptation between clients and the environment. Another component is the active approach which supports the process of using appropriate means to establish medical history and diagnosis, build an evaluation and plan interventions. Social workers are prepared and able to absorb the therapeutic role. Parts of their job are interventions to change the behavior, experience, attitudes and relationships of the individual. Social work helps the individual, by changing the client, it develops the ability to cope, increase or support the potential of adaptation and independent living, improves quality biopsychosocial functioning, stabilizes personality experiences and behavior, balances and improves the quality of relationships with the social environment. The profession of social worker promotes social change, problem-solving and empowerment of the individual to improve well-being. The social worker intervenes in those aspects where people interact with the environment. The bases of social worker practice are the principles of human rights and social justice. Many of the roles performed by the social worker are the same as those of other mental health professionals. What distinguishes the social worker from others is his role in building partnerships between professionals, careers and family members; working with the community to create supportive environments for patients, advocate for appropriate services, treatments and resources for them, change social policies on issues of poverty, employment, housing and social justice, and support prevention programs. The study examines both the opinion of social workers who are the real providers of social services in our country and the persons with mental health disorders and/or their relatives, the usefulness and effectiveness of existing process services to integrate people with mental disorders mental disorders over the age of 18th.The two main concepts of this study are the role of the social worker in mental health and supported homes. A good part of this study will also focus on how the social worker's service affects mental health and supported lodges. The novelty of this study will shed light on the integration process, the degree of success of reforms in the social spheres in our country and the stages achieved in this process. It will also highlight the advantages and disadvantages of services and policies designed for this target group.

The voice of the beneficiaries that will come through the answers provided by the questionnaires will identify current and urgent problems and on their basis will be issued the recommendations of the study.

\subsection{History of Elbasan Psychiatric Hospital}

The psychiatric hospital of Elbasan was opened in 1963, and after it was opened the psychiatric hospital of Shkodra. The psychiatric hospital of Elbasan was opened in order to treat neurological and psychiatric diseases. Thousands of patients from different districts of Albania have been treated at this Hospital over the years. Elbasan's Psychiatric Hospital is a regional and largest hospital in the Republic of Albania. While before the 1990s the number of hospitalized reached up to 800 people. Currently the number of users has decreased significantly and now there are 310 beds covering a population of about 1363903 inhabitants in a certain area that includes different districts from central and southeastern Albania. 


\section{Material and methods}

\subsection{Service criteria / target group that provides direct or indirect service}

At the mental health service with beds, emergency services are provided through inpatient services, or treatment/rehabilitation services through wards for sub-acute patients, who need longer-term treatment than that provided in the functions of the reception service.

Only individuals who meet the following criteria are referred to the mental health services with beds

- The person is referred by the Community Mental Health Center or the specialist doctor for those areas where there is no MCCM, for more specialized treatment after all existing opportunities for health treatment in the community are exhausted and the necessary treatment is provided only through hospitalization in mental health services, specialized with beds, in accordance with the principles of the least restrictive alternative

- Observation and provision of first aid only in cases for which it is not possible to apply outpatient health interventions (emergency cases)

- Psychiatric counseling is needed in response to requests coming from the emergency of general hospitals or requests coming from other hospital wards, which cannot be addressed to KCSM

\subsection{Service objectives}

This service is intended to serve individuals in need of hospitalization in order to better assess, treat and alleviate acute psychiatric symptoms which limit / disable the individual to function in the community for a certain period of time. As hospitalization is the most restrictive of mental health services, its purpose is to stabilize acute symptoms so that the patient can return to the community as soon as possible or to other services provided at that level. Individuals who, in addition to mental health disorders, also suffer from other disorders such as mental retardation or substance abuse, are admitted to our service only if hospitalization is necessary to treat the mental disorder.

\subsubsection{Admission and exit procedures / criteria}

The random assessment procedures for acceptance include:

- Assessment of the current problems of the individual or his needs by the multidisciplinary team

- Assessing whether the service can provide services that address the needs of the individual.

- As well as possible referrals.

\subsubsection{First evaluation criteria}

The first assessment, at the time of hospitalization in mental health service beds is carried by the psychiatrist of the ward where the patient is admitted, who also makes the admission diagnosis, as well as other members of the multidisciplinary team (psychologists, social workers) within the first 24 hours of its laying,

After several intensive assessments by the team within the first days of hospitalization, the clinical diagnosis is made no more than 7 days after his hospitalization.

\subsection{Service functions}

A - This service is designed to provide treatment for individuals with acute psychiatric problems and is the most intensive and restrictive service for individuals. This service offers:

- Medical and psychological diagnostic procedures

- Observation of the progress of the disease

- Different treatment modalities, such as medication, psychotherapy, counseling, occupational therapy, etc.

- Appropriate treatment and health care

- Accommodation and food according to standards

B - Bed service provides the safest and most respectful services for the individual, including, based on the least restrictive alternative:

- Using the least restrictive methods and the most appropriate methods for the patient,

- Promoting collaborative skills to minimize risky behaviors towards oneself and others, 
- Providing the opportunity to include activities as meaningful as possible for the patient,

- Making decisions on the intervention plan in close cooperation with the patient (informed approval).

- In the case where it is impossible to avoid procedures such as restriction and isolation, service ensures that this is the last option left, and that the procedures are carried out according to the standards and skilled people.

C - All patients have an individual treatment and rehabilitation plan which is made by members of the multidisciplinary team, as well as an outpatient plan which is discussed with the patient and his/her family to reflect needs.

D - The hospitalization of a person with mental health disorders in mental health service with beds cannot be used in any case, for the purpose of examining the ability to work.

E - All new patients are admitted to the admission ward, when the evaluation, diagnosis and treatment of acute symptoms are performed in function of the service objectives and the patient's return to the community as soon as possible. Only the psychiatrist has the right to transfer the patient from this service to other services, or to decide on his discharge from the hospital.

F - New patients are never admitted directly to sub-acute service. In this pavilion are transferred. Only patients from the reception ward who need more time for hospitalization, or for residents of residential centers whose health condition has deteriorated. Patients can leave this ward directly for home. Visits by a specialist are less frequent for patients in this service and the occupational therapist / social worker makes an individual rehabilitation plan for this treatment phase.

\subsection{Rehabilitation services}

\subsubsection{Day centers}

- 2 day care centers, "RILINDJA" and "TULIPAN", are coordinated rehabilitated services of mental health services with beds, provided at the hospital level, which provide activities for recovery of lost abilities, development of new skills, educational and psycho-educational services, pre-vocational training for individuals with chronic mental health disorders usually on a part-time basis during the day, practically 09:00-16:00. These services aim to rehabilitate, support chronic patients to identify and develop their strengths, in order to empower them to function as an independent individual and in interaction with society and the community in its future, outside the services with beds.

These services refer mainly to users of mental health services with beds, who are chronicled by long-term stay in the premises of the institution. The users of these day care centers have an affected function in areas such as: employment, income management, social networking, daily life activities, etc.

Referrals of patients to this service are carried by doctors, psychologists, social workers and head nurses of each ward. The selection of these patients is made by the head of the day care centers, in accordance with the realization of the purpose of the service provided by these centers, their capacity and the type of activities planned in them. The service provided at the day care center coordinates pre-designed individual care plans.

Service objectives

- To train patients in achieving the optimal level of functioning;

- To enable patients to live independently;

- Empower patients to understand and manage their disease as efficiently as possible;

- Promote the involvement of family members in care.

Main functions of the service

Day Care Centers provide the following services, but are not limited to, such as Social Skills Development Services, which include:

- Skills of living in the community, such as house maintenance, shopping, cooking, use of public transport, income management;

- Skills for personal care, such as personal hygiene, health care, medication management, etc.;

- Ability to create social relationships;

- Leisure management. Educational services, which include psycho education and education in areas of interest. 
- Pre-vocational services aimed at developing good habits related to employment and participation in various occupational activities.

These services are provided through the following interventions:

- Formulation of individual care plans - As part of the biopsychosocial management of all patients, individual care plans should be developed. The plan is multidimensional, includes needs for education, disorder management, vocational rehabilitation and cognitive rehabilitation;

- Psycho education - is provided continuously in order to inform, support and train the patient in situation management strategies. It contains at least components related to providing information on the disorder and its management, the first signs of relapse, crisis management, medication and side effects.

- Working with families - aims to educate and support the family in order to understand and manage the disorder as effectively as possible, as well as to promote the living in them of patients.

- Social skills training - includes supporting the patient in regaining basic social skills, often lost due to institutionalization, such as personal hygiene care, communication skills, building social relationships, money management, cooking, etc.

- Day care centers are equipped with information and audiovisual tools, as well as other tools needed to perform the above activities.

\subsubsection{Supported houses}

The supported homes are aimed at rehabilitating persons suffering from mental disorders and display inability to cope with life after discharge from hospital. Some of them are:

- Providing the service only for chronic patients hospitalized for years in the psychiatric hospital of Elbasan.

- $\quad$ Providing a good quality service (not just good environmental conditions).

- Implement the Mental Health and Human / Patient Rights Reform.

- Improving personal competencies / learning lost skills.

- Integration with the community environment and restructuring of the way of life.

- Support and support of users (residents).

- Empowering users (self-expression / self-selection / self-determination).

- Encourage users to express this empowerment everywhere / outside the home

- Reconstruction of quality relationships with the user support network (family, friends, etc.)

- Rehabilitation of users (being contributors / citizens) in the surrounding / relevant community.

\subsection{Social work in mental health in Albania}

The social worker works with patients individually, with family members, protects patients' rights, uses resources by favoring the individual's connections with other organs. The social worker is an important part of the multidisciplinary team and works by recognizing the demands and needs of patients, investigating the case and focusing on the socioenvironmental situation. The social worker works by encouraging the individual to explore the outside world, utilizing and supporting in various sources charitable institutions, unions, family support in order to integrate them into society. Also performs various activities for the benefit of individuals, groups and families to resolve situations.

The purpose of social work interventions is to maintain and strengthen the health of clients by working with their social problems and in particular, improving their relationship for adaptation between clients and the environment. A component of clinical social work is the active approach which supports the process of using appropriate tools to establish medical history and diagnosis, build an evaluation, and plan interventions.

\section{Results and discussion}

The main purpose of data analysis was to review and organize the amount of information obtained from conducting interviews so that the topics and interpretations that would emerge from this process address the questions raised at the beginning of the study. The important topics and concepts that emerged from reading the interviews were noted on a piece of paper. In some special cases the interviews were coded and analyzed at the time of their collection, but in most cases their analysis and transcription began after the interviews were completed. 
The transcripts were also accompanied by participants' signs or body language reflecting nonverbal interactions, as well as pauses, off-topic debates, and momentary interruptions due to outside interference [4]. In some cases it was necessary to rewrite the transcripts verbatim to give as much meaning as possible to the context of the conversation.

Data management is done manually. After gathering the necessary information from all interviews, the researcher has identified the units of meaning by coding and interpreting the data continuously;

\subsection{Interviews with social workers and occupational therapists of Day Care Centers}

According to the information obtained from the interviews with social workers who practice their profession, we currently learn that a total of 4 social workers are employed, where 3 three of them are focused on the psychiatric hospital of Elbasan and only 1 social worker covers services for individuals with mental problems in the Center. From the interview conducted with these employees and from their daily practice we conclude that the focus of social work on mental health is assessment, diagnosis, treatment and prevention of mental illness.

The mission of the social worker in mental health is to increase and empower patient well-being by:

- Utilize all possible resources

- Raise and involve them in treatment, decision making and rehabilitation.

- Provide standardized professional service based on fundamental human rights and freedoms and mental health law.

\subsection{Social worker principles in mental health}

- Equal and non-discriminatory treatment of persons with mental health disorders in order to respect physical integrity and human dignity [5];

- Providing health care for people with mental health disorders in a less restrictive environment, mainly at the community level to avoid as much as possible displacement from the family environment and to facilitate social integration and rehabilitation.

- Creating facilities for these people and their families in order to include them in social life.

- Providing care for people with mental health disorders by multidisciplinary teams that respond in a complex way to medical, psychological, social and rehabilitation needs.

- Exercising the rights of persons with mental health disorders in accordance with international acts, ratified by the state.

\subsection{Listed below are some of the services offered by the social worker in the mental health institution.}

- Psycho-social assessment in the system

- Intervention in crisis situations

- Visit to the settlement

- $\quad$ Structured therapeutic-rehabilitation intervention

- Monitoring of psychiatric somatic therapy

- Clinical meetings on team cases

- Individual psycho-emotional counseling

- Individual psycho education with the patient

- Group psycho education with the patient

- Individual psycho education with family

- Group psycho education with family members

- Counseling / support groups with family members

- Facilitation of self-help groups

- $\quad$ Support in meeting basic needs

- $\quad$ Structured preventive and promotional activity on mental health

- Educational-recreational activities

- $\quad$ Case management

- Advocacy

- Information / referral 


\subsubsection{Activities organized by the social worker in the psychiatric hospital, in day care centers and supported lodges}

- Psycho-educational activities (on mental illness, sexually transmitted diseases, raising and caring for children, etc.

- Various formative activities such as painting, music, carpentry, pottery, embroidery, knitting, etc.

- Fun and socializing activities in the center or in the community, comedy shows in the center, giving coffee or tea, celebrating the birthdays of all patients $1 \mathrm{~h} /$ month, going out in an organized way in the bar, cinema day, Catholic Mass day, picnics on the outskirts of the city, excursions outside the city, etc.

- various professional trainings, fun games, football matches.

\subsubsection{Respondents' opinions on the importance of the functioning of the multidisciplinary team}

- The multidisciplinary team is very important because it gives and exchanges ideas and opinions which aim to rehabilitate the patient as soon as possible.

- The multidisciplinary team builds the individual plan of appropriate interventions according to the specifics of each patient where according to this plan each professional programs the intervention services.

- The way the multidisciplinary team works greatly affects the effectiveness of the services provided to persons suffering from mental health disorders.

- Symptom assessment, treatment, rehabilitation, and integration of people with mental health problems depend heavily on how the multidisciplinary team interacts.

\subsubsection{The importance of supporting family members who have mental health problems}

- It is true that family education and the way a family is treated means a lot in its further development.

- Many mental disorders have their origins in early childhood and it is the hidden traumas that accompany and affect the identity and personality of the individual.

- Every member or every child needs to feel that they belong to a family and society. Must learn to take responsibility. Must learn to cope with the positive and negative aspects of life as well as gain the ability to solve problems

- It also has a big impact on children who have parents with mental health problems, because they find it difficult to understand why their parents with mental health problems act that way different, lonely, and isolated. This can lead them to the development of psychological, behavioral or social problems.

\subsubsection{Interviewers' opinions on social policies drafted by law for this category are sufficient to be effective in your work;}

- There are many policies that are well defined but their implementation in some cases is impossible.

- Target group of people suffering from mental health and belonging mainly to remote rural areas find it impossible to receive medical services, outpatient treatment, as well as other social and economic services due to impossibility and ignorance of where to address .

- Economic assistance is at adequate levels to cope with difficult living conditions.

- There is a lack of initiative to create equal conditions for the employment of these individuals in both state and private institutions.

\subsubsection{Changes that respondents would like to make from their experience and practice in the institution where they work.}

- At first I would rarely add the number of social workers because it is very low in unfair proportion to the number of patients.

- Review of policies addressed to this category of individuals.

- The most rigorous implementation of policies.

- Facilitation policies for families of individuals with mental health disorders.

- Mitigation of some bureaucracies in the out-of-camp procedures of these patients.

\subsubsection{Beneficiaries' knowledge about the profile of the social worker:}

- Yes he has helped me several times.

- He always accompanies me when he withdraws from the disability camp

- Has referred me to some other beneficial and facilitation services for the situation in which I find myself.

- I know that it has a protective function of the interests of people suffering from mental illness.

- I know him because I meet him every day in the ward when we do counseling therapy and various other interventions. 


\subsubsection{Beneficiaries' impressions after meeting with the social worker}

- I felt good after the advice given to me by the social worker.

- After the interventions made by the social worker, I managed to contact my family members as often as possible.

- I felt very good because the last time the social worker accompanied me by ambulance home to meet my parents who are very old.

- My social and economic problems have been significantly alleviated because the social worker directed me to several different organizations and services that helped me a lot.

\subsubsection{Difficulties during the meeting with the social worker}

- In the beginning I did not want to talk about myself and the problems I have but over time I started to ask for a counseling session with the social worker because I felt that when I talked to her the pain was relieved.

- Yes at the beginning I did not want to tell you what was going on in my mind, but then after a few counseling sessions I started working with myself and realizing that those rumors in my head were not true and were there because of my illness.

- No I knew that the social worker protects the rights and interests of patients in the hospital and I asked to speak to him myself as I thought I was being unfairly hospitalized, but after a few meetings with him I began to believe what he was telling me and advised me.

\subsubsection{Beneficiaries' reaction after meeting with the social worker:}

- Confidence in oneself has changed.

- After meeting the social worker, my relationship with my family improved because I thought they did not love me and did not understand me.

- I have started to change behaviors with the people around me; I do not get so irritated anymore.

\section{Conclusion}

- The social worker in mental health does not carry out a medical treatment; it is not based on therapy or medication, but on care.

- The role of the social worker develops best where human desires and aspirations are not hindered; resources and support people are more numerous and do not end when the person leaves the hospital in improved condition.

- Rehabilitation in mental health is a unique process that depends on many factors / it has different intensity and time frame for different people.

- In community services / supported homes, the social and psychological functioning of the subjects is better than in the hospital service, directly affecting the course of the disorder.

- From the perspective of chronic patients, community services are more effective in their efforts to support patients.

- In the hospital service, priority is given to the care and rehabilitation of the disorder.

- Supported housing professionals are more oriented towards rehabilitation concepts and plans compared to hospital professionals. Hospital professionals are more oriented towards medical-nursing service focusing on pathology and psycho-pathological injuries, while community professionals focus on points of view. strengths of the person and the right to choose.

\section{Recommendations}

- Increasing the number of social workers in psychiatric hospitals.

- In addition to the medical-psychiatric plan, there should be plans for well-organized rehabilitation interventions in the wards of the psychiatric hospital.

- To design clearly and formally rehabilitation programs and activities for all chronic patients in alternative mental health services.

- Acknowledging the success and importance of supported homes in Elbasan, efforts should be made to open as many such rehabilitation structures as possible with the aim of de-hospitalizing as many long-term chronic patients in the hospital.

- More specialized support and coordination of social services from other support institutions. 
- Support and develop appropriate procedures and practices for documenting and evaluating mental health services.

- Designing information and prevention projects for the general population regarding the first symptoms of mental health problems.

- Awareness programs to reduce stigma and discrimination against people with mental health problems

\section{Compliance with ethical standards}

\section{Acknowledgments}

I would like to express my sincere gratitude to the supervisor Prof. Monika Bogdanova for the support and proper scientific guidance in the realization of this scientific work, as well as the staff of "Sadik Dinci" Psychiatric Hospital, Elbasan, to provide the necessary conditions for conducting social work analysis.

\section{Disclosure of conflict of interest}

The authors declare that there is no conflict of interest for the presented study.

\section{Statement of informed consent}

Informed consent was obtained from all individual participants included in the study.

\section{References}

[1] Law no. 44/2012 "On mental health". Albania; 2012. Available from https://shendetesia.gov.al/wpcontent/uploads/2018/03/Ligji Nr.44 2012 per shendetin mendor.pdf

[2] The National Steering Committee for Mental Health. The Policy and the Operational Plan for Mental Health Services Development in Albania; 2003. Available from https://shendetesia.gov.al/wpcontent/uploads/2018/03/The Policy and the Operational Plan for M.H 2005 -2010.pdf

[3] Worls Health Organization (WHO). "Mental Health" - Action Plan 2013-2020; 2013. Available from https://apps.who.int/iris/bitstream/handle/10665/89966/9789241506021_eng.pdf;jsessionid=872F794EE7 4BBE0E03C6836626CB9578? sequence $=1$

[4] Banderov A. Clinical social work - development and practice in Bulgaria. E-magazine Social Work (in Bulgarian). 2017; 5(1):45-57.

[5] Krueger AR. Moderating Focus Group. Group Kit Series (Kit 4). Thousand Oaks, Calif.: Sage Publications. 1998; 70. 